\title{
Tactile perception of sequentially presented spatial patterns'
}

\author{
JAMES C. BLISS, STANFORI RESEARCII INSTITUTE AND STANFORD UNIVERSITY \\ HEWITT D. CRANE, STEPHEN W. LINK AND JAMES T. TOWNSEND, STANFORD RESEARCH INSTITUTE
}

Tatile pattern recognition was studied by presenting pairs of alphabctic shapes in rapid succession at the same ana tomical location. the subject being required on each trial to identify both of the patterns. Experimental variables were the duration of cach stimulus and the time between stimuli. Three aspects of the observed interaction were (1) an increase in letter reversals for very short interstimulus intervals; (2) a greater percentage of first-response errors for short-stimulus onset interials and a greater percentage of second-response crrors for long-stimulus onset intervals; and (3) a crossover in the first-and second-response error rates in the range of 100 to $200 \mathrm{msec}$, after the onset of the first stimulus. These results are consistent with some of the temporal properties of models proposed for analogous visual tasks.

Visual recognition of patterns presented sequentially in the same retinal location has been studied by many investigators (e.g., Kolers \& Katzman, 1963; Massa, 1964; Averbach \& Coriel 1961; Eriksen \& Collins, 1965). Phenomena described as temporal interaction, erasure, forward and backward masking, etc., have been observed, and the results from experiments with these phenomena have led to postulated models (e.g., Sperling, 1963) describing the temporal properties of the visual channel. In investigations with patterned tactile stimuli, similar phenomena a re observed, the understanding of which is crucial to attempts at tactile communication and development of a "tactile language."

The experiments reported here were aimed at determining temporal effects in the tactile channel. In these experiments tactile-spatial patterns were presented in rapid succession at the same anatomical location.

\section{Apparafus and Procedure}

The experiments were carried out under control of a computer system that is described in detail elsewhere (Bliss \& Crane, 1964). In this system, a CDC 8090 computer is used to store stimulus patterns and the sequence in which the patterns are to be presented. Figure 1 shows the patterns used in these experiments. These patterns comprise an experimentally developed alphabet that has been found to be convenient for experimentation because the patterns are easily distinguished and learned. To make the results easier to integrate, the relatively more difficult letters $(H, M$, $O, U, Y$, and $Z$ ) were not used (except for the preliminary experiment described here) leaving an effective alphabet size of 20 characters.

The computer was programmed to output these alphabetic shapes in the appropriate temporal sequence.
To present one such shape the computer transmitted a sequence of eight 12-bit words to specially constructed external equipment. Each word represented one row of the spatial pattern to be displayed. The external equipment stored the 96-bit pattern in $8 \mathrm{msec}$. and simultaneously activated the specified tactile stimulators.

The tactile stimulators used were airjets. Each jet was formed from a $\mathbf{0 . 0 3 1 - i n . ~ o u t l e t ~ p o r t ~ a n d ~ w a s ~ a c t i - ~}$ vated by an electromagnet. The air pressure pulse, measured $1 / 8$ in. directly above the airjet outlet, was about $3 \mathrm{psi}$ in these experiments, with a rise and fall time of about a millisecond and an overall pulse width of about $2.5 \mathrm{msec}$. A 200-cps pulse repetition rate was used throughout the experiments, implying that, for example, the stimulators were actually turned on and off 20 times during a presentation time of $100 \mathrm{msec}$.

The advantages of airjet stimulation are that relatively uniform stimulation is produced over nonuniform cutaneous surfaces and stimulator spacing can be made quite small. The stimulator array used is shown in Fig. 2, and the location of the stimulators with respect to the palmar side of the hand is shown in Fig. 3.

Two male and one female college students were used in these experiments.

\section{Preliminary Experiment}

The importance of temporal sequence in tactile perception of alphabetic shapes was first noted in an experiment in which a subject was presented with random letters in two different temporal sequences but at the same average rate of letter presentation. In the first sequence, every 0.9 sec. a letter (chosen at random) was presented for $0.3 \mathrm{sec}$. and was followed by a $0.6-\mathrm{sec}$. rest. The subject had to respond in the $0.6 \mathrm{sec}$. off-time between the end of one letter and the onset of the next. In the second sequence, sets of three random letters were presented in rapid succession during $0.9 \mathrm{sec} .$, each letter being on for $0.3 \mathrm{sec}$. In the following $1.8 \mathrm{sec}$. the subject had to name all three letters. He then received three more letters, followed by $1.8 \mathrm{sec}$. off-time, and so on. Four sessions of $1 \mathrm{hr}$. each were run, two sessions with each temporal sequence. During each session there were four tests, separated by rest and practice periods. Each test consisted of 81 letters. The results of this experiment are shown in Fig. 4.

Under the second-sequence conditions, the performance was extremely poor. The fact that the subject missed almost every middle letter of each triplet sug- 

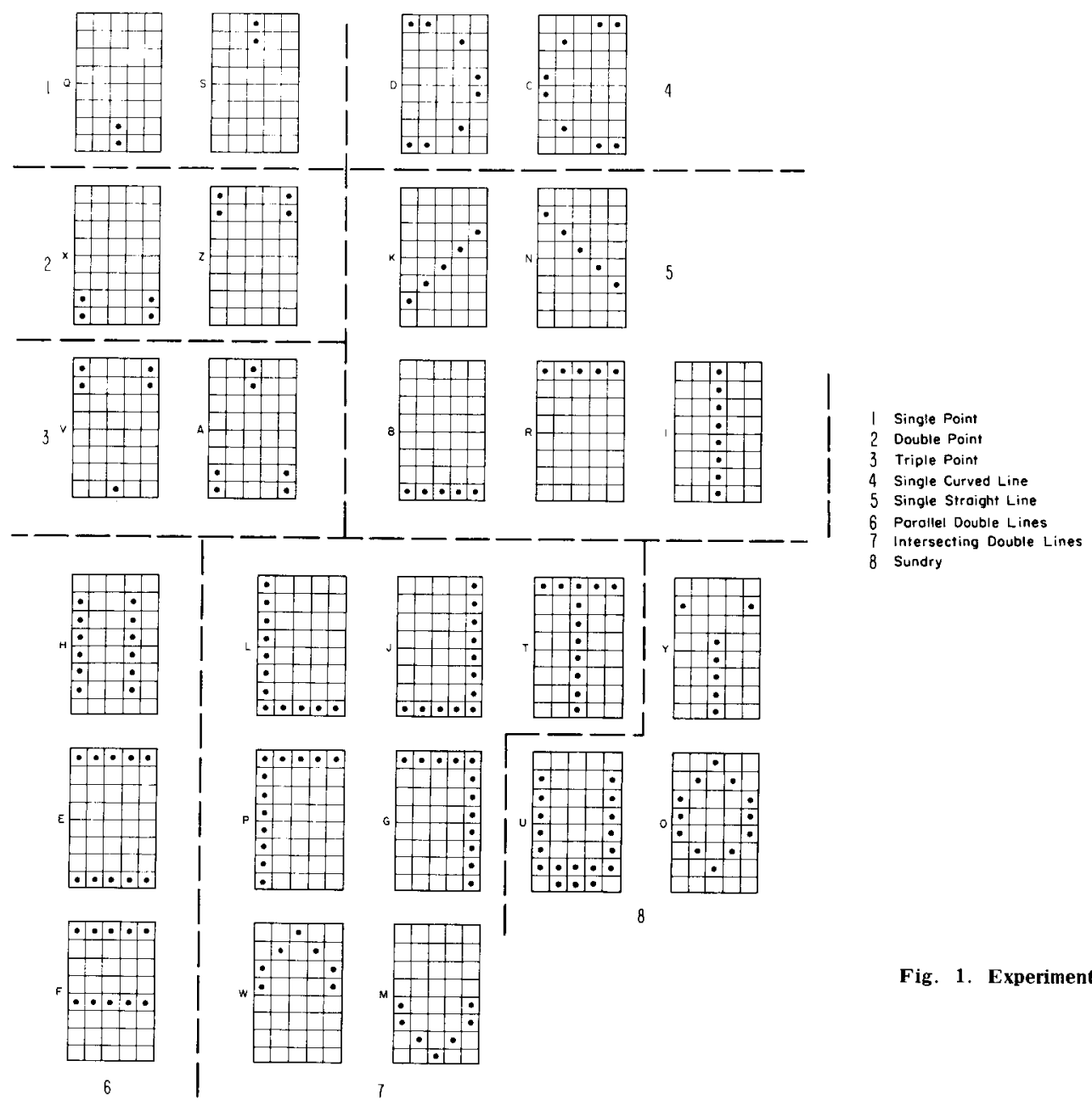

Fig. 1. Experimentally developed alphabet.

gested that some type of masking was in part responsible for the poor performance. It was also found that by counting a response correct when it was simply in the

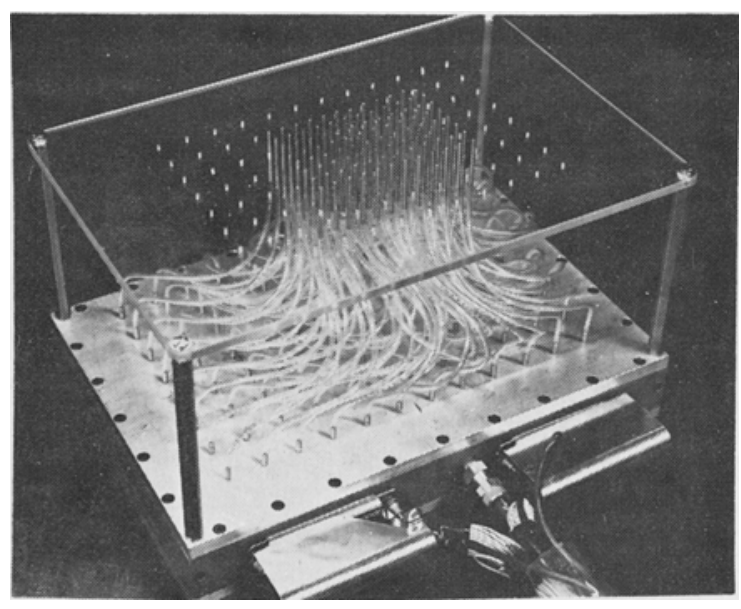

Fig. 2. Tactile stimulator array.

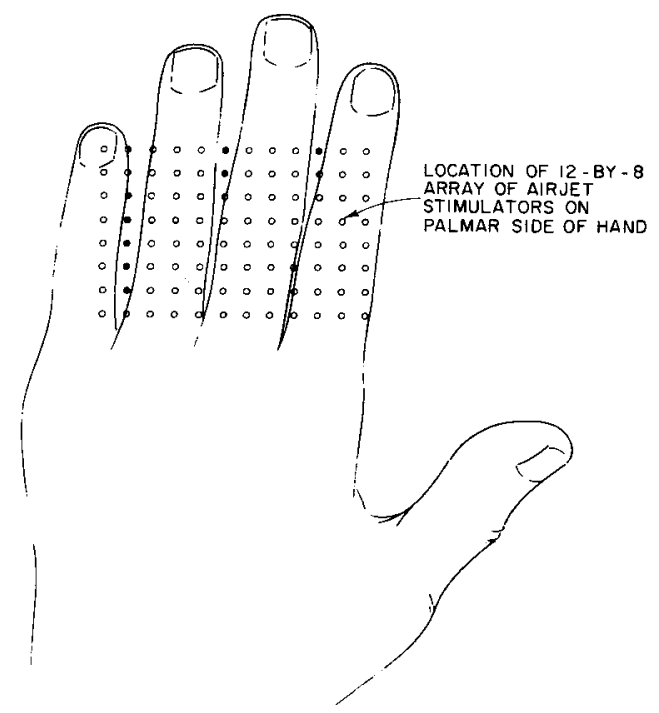

Fig. 3. Position of airjet array about $1 / 4$ " below the palmar surface of the hand. 


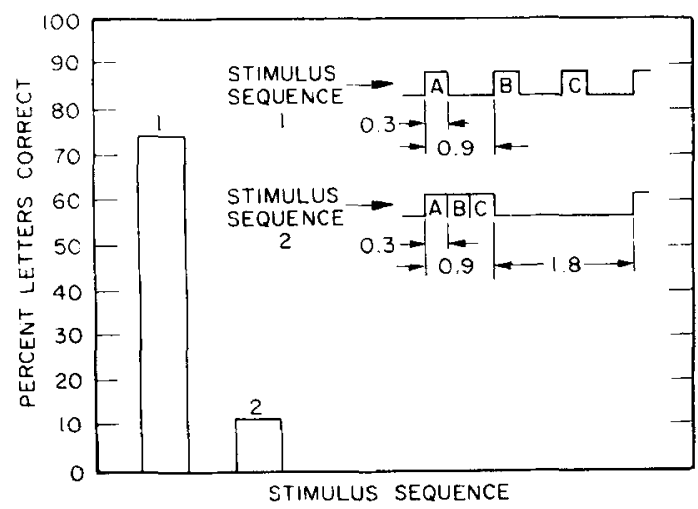

Fig. 4. Letter recognition accuracy for two temporal sequences.

wrong sequence, the subject's performance (after correction for guessing) was essentially doubled. Thus, by counting KJP in response to PJA as two letters correct (the $P$ and J) instead of just one (the J), the subject's accuracy-corrected for guessing-increased from 11 percent to 20 percent. This is in agreement with the results reported by Kolers and Katzman (1963) for a visual experiment in which the subject was asked to name English letters sequentially presented in groups of three at letter rates approximately twice as fast as the ones used in the tactual experiments described here. Kolers and Katzman determined that this kind of letter reversal was a common phenomenon. This similar finding, for both touch and vision, implies that the major problem in the triplet experiment may not be at a peripheral neural level, since more letters are recognized than indicated by the performance scores, but their sequential ordering is not being preserved.

\section{Doublet Experiments}

The preliminary experiment led to a series of sessions in which random pairs of alphabetic characters were given each of the three subjects according to the temporal sequence shown in Fig. 5. Following each doublet presentation, a subject responded at his own rate. His responses were then typed on the on-line typewriter by the experimenter, thus automatically activating the next stimulus sequence. The subjects were very well practiced in this task, each subject having at least $10 \mathrm{hr}$. of training before the tests began.

During each $1-\mathrm{hr}$. test session, four separate tests were given, with rests between. Each test consisted of a presentation of 66 pairs of equally probable random letters. The tests with Subjects $R$ and $K$ were run first, using a predetermined set of time intervals $\left(T_{0}, T_{1}\right)$; the tests with Subject Ke were run later. The results from Subjects $\mathrm{R}$ and $\mathrm{K}$ were used to select a better set of time intervals $\left(T_{0}, T_{1}\right)$ for Subject Ke.

In one set of test sessions, $T_{1}$ (the off-time between the letters of each pair) was held constant at $22 \mathrm{msec}$, and $T_{0}$ (the on-time of each letter) was varied from 100 to 400 msec. for Subjects $R$ and $K$, and from 50 to $300 \mathrm{msec}$. for Subject $\mathrm{Ke}$. The results are shown in Fig. 6 , in which the percent of errors on the first response and the percent of errors on the second response are plotted separately as a function of $T_{0}$. For all three subjects, the error rate decreased as $T_{0}$ increased, more first-response errors occurring with values of $\mathrm{T}_{0}$ less than 100 msec., and more second-response errors occurring with values of $\mathrm{T}_{0}$ greater than 200 msec. The crossover occurred for $T_{0}$ between 100 and $200 \mathrm{msec}$. In other words, for short letter durations, there seemed to be more interference of the second letter with perception of the first, while for longer durations the reverse seemed to be true.

In a second series of test sessions with Subjects $R$ and $K, T_{0}$ was held constant at 100 msec. while $\mathrm{T}_{1}$ was varied from 22 to $300 \mathrm{msec}$. For Subject $\mathrm{Ke}$, $T_{0}$ was held constant at $50 \mathrm{msec}$. while $T_{1}$ was varied from 22 to $400 \mathrm{msec}$. These results are shown in Fig. 7, where both first- and second-response errors are independently plotted as a function of $T_{1}$. Again, there is a crossover between first- and second-response errors, first-response errors being more prevalent for short $T_{1}$ intervals and second-response errors more prevalent for longer $\mathrm{T}_{1}$ intervals. Each of the error rates decreased with $\mathrm{T}_{1}$ to values of about 10 percent.

In a third sequence of tests with Subjects $R$ and $K$, the time between letter onsets was kept constant at 400 msec., while $\mathrm{T}_{0}$ was varied from 100 to $400 \mathrm{msec}$. These data are shown in Fig. 8, where total errors are plotted as a function of $T_{0}$. These curves indicate a minimum in error rate for values of $T_{0}$ between 200 and 300 msec. implying that a period of no stimulation for about $100 \mathrm{msec}$. between letters is beneficial.

A final result apparent from the data is that letter reversals occur only for small values of $T_{0}$ and $T_{1}$. Letter reversals accounted for about 4 percent of the

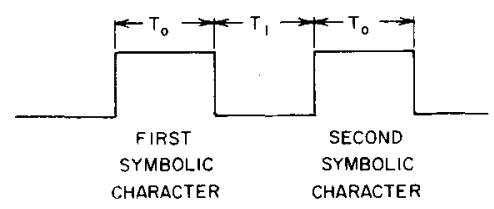

Fig. 5. Timing arrangement for sequential presentation of the pair of alphabet characters. 


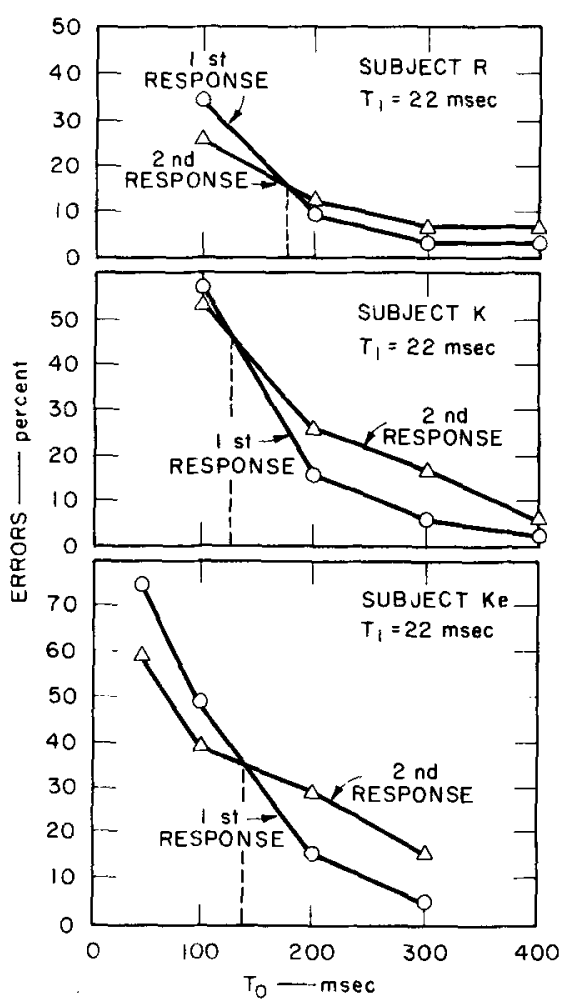

Fig. 6. Percent errors as a function of stimulus on-time with the between-stimuli interval held constant.

errors for $T_{I}+T_{0}$ less than $150 \mathrm{msec}$. (for Subjects $K$ and $\mathrm{Ke}$ ) and accounted for a negligible percentage of the errors for $T_{1}+T_{0}$ greater than $150 \mathrm{msec}$. Subject $R$ had a total of only five letter reversals in all of the sessions; these reversals were all for $T_{1}$ equal to 22 msec. and $\mathrm{T}_{0}$ between 100 and $300 \mathrm{msec}$.

\section{Discussion}

Since the subjects had to identify both of the temporally separated stimuli in each of the doublet trials, these experiments may be considered as a pattern masking study in which both forward and backward masking phenomena are involved simultaneously. That there should be interference between two stimuli presented too closely in space or time is, of course, not unexpected, since any system, including the human nervous system, has limited resolution. However, the following three aspects of the interaction' results reported here are worth special mention: (a) an increase in letter reversals for very short interstimulus intervals; (b) a greater backward masking effect for small values of $T_{0}$ and $T_{1}$, and a greater forward masking effect for longer values of $T_{0}$ and $T_{1}$; and (c) a crossover in (b) occurring in the range of 100 to $200 \mathrm{msec}$. after the onset of the first stimulus.

While the picture is far from complete, many investigators have suggested models of perception based

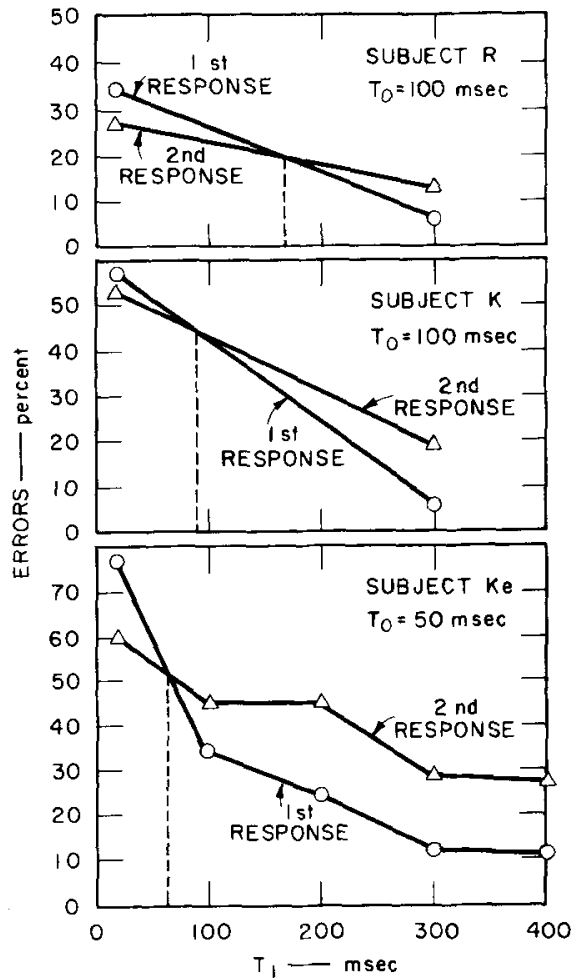

Fig. 7. Percent errors as a function of the betweei-stimuli interval with the stimuli on-time held constant.

on input quantization of time. Eriksen (1966), for example, suggests that the visual system sums the luminance from two or more successive stimulations distributed within a brief time interval of the order of $100 \mathrm{msec}$.

Although there have been some well stated objections to models involving constructs like "epoch," "read-in time," and "erasure," such models can help structure thinking on masking and interference phenomena, even though these constmucts are oversimplifications. In particular, it is worth considering whether such models can be applied to tactile memory tasks. For example, in a model proposed by Sperling (1963) for visual memory tasks, there is a read-in interval of roughly 50 to $100 \mathrm{msec}$. Stimuli occurring wholly within this interval tend to summate and superimpose. Normally without interfering stimuli, the read-in period is followed by a short-term storage, processing, and readout interval lasting perhaps as long as several seconds. However, a second stimulus occurring immediately after the read-in interval of the first stimulus, just before or during the short-term memory read out, may tend to initiate a new read-in interval and cancel or replace the first stimulus before it is read out. With still further separation, the two stimuli occur in separate "memory epochs," and their mutual interference is reduced. Thus, according to this model there are at 


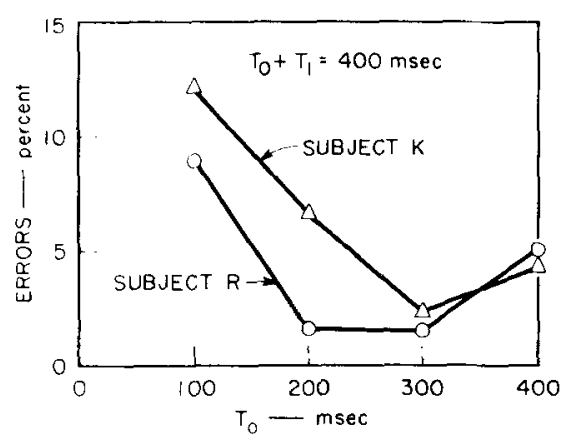

Fig. 8. Percent errors as a function of stimulus on-time with the stimuli-onset interval held constant.

least three intervals of concern: (a) a summation interval of 50 to $100 \mathrm{msec}$; (b) an interval immediately after (a), in which a second stimulus may tend to replace a first stimulus; and (c) a later interval of little interference.

In terms of such a model, whether there should be more first- or second-response errors in the experiments reported here is determined by which of these intervals is involved. Of course, for simultaneous presentation of two patterns, the number of errors on the first stimulus must equal the number of errors on the second stimulus. Presumably this would also be true for two stimuli occurring wholly within interval (a) above. Since the curves of Figs. 6 and 7 show no tendency to come together again for the short values of $\mathrm{T}_{0}$ and $T_{1}$, the interpretation would be that the minimum values of $T_{0}$ and $T_{1}$ employed in these experiments were sufficiently long that in most cases only conditions (b) and (c) mentioned above occurred. This is also suggested by the fact that the percentage of reversal errors never became very great. The fact that the percentage of reversals increased somewhat above a chance level for the shortest times employed indicates that in a few cases interval (a) phenomena were involved.

According to this interpretation, then, when the second letter occurs immediately after the read-in time, the first letter may tend to be cancelled or replaced, thereby producing more first-response errors. With further temporal separation, the first letter gets safely tucked away in immediate memory before the second letter is presented, the reby reducing the first-response errors. The first- and second-response cross-overs shown in Figs. 6 and 7 suggest that the interval in which the second stimulus tends to replace the first is from about 75 to $200 \mathrm{msec}$.

Also consistent with a tactile epochal model are the results from tactile apparent-motion studies. These apparent-motion phenomena occur strongly for stimuli temporally separated by 50 to $150 \mathrm{msec}$.(e.g., Kotovsky \& Bliss, 1963; Sumby, 1965), which would place the stimuli in adjacent read-in intervals. When the stimuli are separated by one or more memory epochs, the system should be able to resolve the ambiguity, and the perception should be that of two spatially separate stimuli instead of one moving stimulus.

A number of tactile neurophysiological experiments have indicated response phenomena involving intervals of the order of $100 \mathrm{msec}_{\circ}$, which are suggestive of underlying mechanisms for masking or erasure. In a pertinent experiment, Towe and Amassian (1958) recorded action potentials from single cortical cells in somatosensory area 1 of rhesus monkeys (Macaca mulatta). On stimulating the palmar surface of the digits and hands, they found that the evoked discharge of 40 of the 110 units encountered could be prevented by a prior or simultaneous stimulation at a nearby point, even when the nearby point, stimulated alone, would not fire the unit. The duration of this inhibitory effect was as long as $100 \mathrm{msec}$, and it was followed by a facilitation period. Presentation of the efficacious stimulus up to $2 \mathrm{msec}$. prior to the ineffective "conditioning" stimulus resulted in complete inhibition of discharge in only three of the units studied.

This inhibitory phenomena followed by a period of facilitation is also found in compound cortical-evoked potentials with cutaneous stimuli. In a review of this work, Uttal (1965) points out that several investigators have found that components of the second of two evoked responses were diminished in size in the $100 \mathrm{msec}$. following the initial stimulus, and that these temporal inhibitions led to vast deviations from a simple additive process.

On a more peripheral level, Lindblom (1965) found long-duration inhibition phenomena in dorsal root units of monkeys as a result of mechanical stimulation of the distal glabrous skin. By means of threshold studies, he demonstrated that each nerve impulse is followed by a relative refractory period which lasts more than $100 \mathrm{msec}$. Repetitive discharge delayed the recovery further and produced a cumulative increase in threshold which rendered sustained firing at frequencies about 60 impulses per second difficult or impossible.

While the mechanism underlying forward masking may be the physiologically observed inhibition, it does not appear likely that this could account for the backward masking. However, backward masking could result from some process associated with the facilitation stage following the inhibition phase observed in neurophysiological responses. This facilitation stage occurs at about the same time, with respect to the onset of the first stimulus, as the period in which fewer second-response errors occurred in the tactile experiments reported here.

Whether these and other findings will eventually "fall into place" cannot be foreseen. At this point, more direct evidence is needed before any model of information processing in the tactile system can be considered more than crude and speculative. 


\section{References}

Averbach, E., \& Coriell, A. S. Short-term memory in vision. Bell le' $h, J ., 1961,40,307-328$.

Bliss. J. C., \& Crane, H. D. A computer-aided instrumentation system for studies in tactual perception. Proc. of the 16th Annual NAECON Conf., Dayton, Ohio, 1964. Pp. 375-384

Eriksen, C.W. Temporal luminance summation effects in backward and forward masking. Percept. \& Psychophys., 1966, 1 (3), 87-92.

Eriksen, C. W., \& Collins, J. F. Reinterpretation of one form of backward and forward masking in visual perception. $J$. cap. Psuchol., 1965, 70, 343-351.

Kolers, P. A., \& Katzman, M. T. Naming and reading sequentially presented letters. Presented to the Psychonomic society, August 20. 1963 .

Kotovsky, K., Bliss, J.C. Tactile presentation of visual information. IEEE Trans. on Medical Electronics, 1963, MIL-7, 108-113.

Lindblom, V. Properties of touch receptors in distal glabrous skin of monkey. J. Neurophys., 1965, 128, 966-985.

Massa, R. J. The role of short-term visual memory in visual information processing. Symposium or models for the perception of speech and visual form. Boston, Mass., 1964.

Sperling. G. A model for visual memory tasks. Hum. factors, 1963, 5. $19-32$.
Sumby, W. II. An experimental study of vibratactile apparent motion. Res. Bull. Amer. Foundation for the Blind, 1965, 9, 71-101. Towe, A. L., \& Amassian, V. E. Patterns of activity in single cortical units following stimulation of the digits in monkeys. $J$. Neturuphys., 1958, 21, 292-311.

Uttal, W. R. Do compound evoked potentials reflect psychological codes. Psychol. Bull, 1965, 64, 377-392.

\section{Nate}

1. The work reported in this paper was supported in part by the Research and Technology Division of the Air Force Avionics Laboratory, Aeronautical Systems Division, under Cruntract AF 33(615)-1099 with Stanford Research Institute, and in part by the National Institute of Neurological Diseases and Blindness under Grant NB 06412-01 with Stanford University. The airjet tactile stimulators used in this study were developed at Stanford Research Institute under Contract NAS 2-1679 with Ames Research Center, National Aeronautics and Space Administration, Moffett Field, California.

(Received the Editorial Office April 9, 1966.) 